Théologiques

Théologiques

\title{
Vieillir et croître à travers les déclins, un défi spirituel avant tout
}

\section{Raymonde Cossette et Jacinthe Pepin}

Volume 9, numéro 2, automne 2001

Sens et spiritualité dans les pratiques professionnelles

URI : https://id.erudit.org/iderudit/007295ar

DOI : https://doi.org/10.7202/007295ar

Aller au sommaire du numéro

Éditeur(s)

Faculté de théologie de l'Université de Montréal

ISSN

1188-7109 (imprimé)

1492-1413 (numérique)

Découvrir la revue

Citer cet article

Cossette, R. \& Pepin, J. (2001). Vieillir et croître à travers les déclins, un défi spirituel avant tout. Théologiques, 9(2), 47-67. https://doi.org/10.7202/007295ar d'utilisation que vous pouvez consulter en ligne.

https://apropos.erudit.org/fr/usagers/politique-dutilisation/ 


\title{
Vieillir et croître à travers les déclins, un défi spirituel avant tout
}

\author{
Raymonde COSSETTE \\ inf. Ph.D, Département des soins infirmiers \\ Cégep du Vieux Montréal
}

Jacinthe PEPIN

inf. Ph.D, Faculté des sciences infirmières

Université de Montréal

En Occident, les progrès combinés de la médecine, de la technologie, de l'hygiène et des co nditions de vie ont contribué à une élévation considérable de l'espérance de vie. Pour plusieurs aînés, la courbe de l'espérance de vie en bonne santé n'ayant pas suivi celle de l'espérance de vie, les années arrachées à la mort s'avèrent être des années d'incapacité. En regard des valeurs privilégiées par les sociétés occidentales (jeunesse, beauté, autonomie, productivité, performance), cette période de déclins apparaît souvent vide de sens et sans aucun attrait ${ }^{1}$.

Pourtant, selon les données épidémiologiques de la psychiatrie, la majorité des personnes âgées qui vivent en hébergement, là où les pertes se multiplient, ne présentent pas de sentiments dépressifs. Des chercheurs se disent même étonnés de la sérénité générale manifestée par les résidents âgés en centre d'accueil et rapportent que les aînés en perte d'autonomie se donnent davantage de buts et sont plus ouverts sur l'avenir que leurs pairs plus autonomes ${ }^{2}$. Ces constats laissent entrevoir la possibilité que, dans l'expérience des déclins de la

1. M.A. KimBLE, "Aging and the Search of Meaning ", dans J.J. SEEBER, dir., Spiritual Maturity in the Laters Years, New York, The Haworth Press, 1990, p. 111-129 ; H.R. MoOdY, Abundance of Life, New York, Colombia University Press, 1988.

2. R. L'ÉCUYER, Le développement du concept de soi de l'enfance à la vieillesse, Montréal, Les Presses de l'Université de Montréal, 1994 ; L. BOUFFARD, 
vieillesse, il y ait des gains peu visibles que la recherche actuelle ne met pas en évidence.

Des auteurs provenant des sciences humaines et des sciences de la santé ont proposé des théories selon lesquelles la croissance se poursuivrait tout au long de la vie. Selon ces théoriciens, la vieillesse serait une étape propice pour s'ouvrir à une expérience de transcendance intra, inter, ou transpersonnelle, laquelle incite à analyser la réalité à partir d'un cadre de sens élargi. Tornstam a introduit le concept de "gérotranscendance " pour qualifier cette caractéristique du développement adulte ${ }^{3}$.

Par ailleurs, philosophes et théologiens ont défini la spiritualité comme une aspiration vers la transcendance. Ce rapprochement entre les humanités et la science suggère que les distinctions entre croissance et spiritualité deviennent de plus en plus ténues à mesure que la personne vieillit. C'est donc à la spiritualité en tant qu'expérience de sens et de croissance alors que les déclins s'accumulent qui se sont intéressées les auteures de cette étude. Afin de laisser les participants âgés discourir librement sur leur expérience de croissance, une méthode de recherche qualitative la moins directive possible, la méthode phénoménologique, a été choisie.

Le but de l'étude était de décrire phénoménologiquement l'expérience de croissance à travers les déclins durant la vieillesse. L'expérience de croissance est définie comme la perception de changements positifs identifiables reliés à la vieillesse tels que décrits par les répondants âgés. La recherche voulait répondre à trois questions :

1. Quelles sont les caractéristiques essentielles de l'expérience de croissance de personnes âgées vivant plusieurs déclins ?

2. Quelle est la structure générale de l'expérience de croissance de personnes âgées vivant plusieurs déclins ?

3. Quelle part la spiritualité occupe-t-elle dans l'expérience de croissance de personnes âgées vivant plusieurs déclins ?

É. BASTIN et S. LAPIERRE, " Extension temporelle des buts et âge chronologique au cours de la vieillesse ", La revue canadienne du vieillissement / Canadian Journal on Aging, 7 (1991) p. 417-430.

3. L. TORNSTAM, « Gerotranscendence : The Contemplative Dimension of Aging ", Journal of Aging Studies, 11 (1997) p. 143-154. 


\section{1. État de la question}

Dans une perspective interdisciplinaire, le dépouillement des écrits a poursuivi l'objectif de cerner le phénomène de croissance durant la vieillesse et sa relation avec le phénomène de spiritualité ou de transcendance, à partir de perspectives issues de la philosophie, de la théologie, des sciences humaines et de la santé et des sciences sociales.

\subsection{Croissance et spiritualité :}

perspectives philosophiques et théologiques

La réponse à la troisième question exigeait de définir le sens qui serait donné au concept de spiritualité dans cette étude. Pour ce faire, les conceptions de la spiritualité d'auteurs incontournables, ceux dont les noms apparaissent aussitôt qu'un écrit scientifique sur la spiritualité retourne aux sources philosophiques et théologiques, ont été étudiées. En psychologie et en psychiatrie, ce sont les thèses de Bergson et de Tillich qui sont le plus souvent évoquées, alors que les sciences infirmières se réfèrent davantage à Buber, Marcel et Teilhard de Chardin. Des écrits de chacun de ces auteurs ont donc été examinés pour en dégager une définition de la spiritualité.

\subsubsection{L'élan vital selon Henri Bergson}

Bergson voit "dans l'évolution entière de la vie sur notre planète une traversée de la matière par la conscience créatrice, un effort pour libérer, à force d'ingéniosité et d'invention, quelque chose qui reste emprisonné chez l'animal et qui ne se dégage définitivement que chez l'homme ${ }^{4}$ ". L'hypothèse de Bergson est que la vie est entraînée par un élan irrésistible vers une efficacité de plus en plus grande. Cet élan qui cherche à se libérer des entraves de la matière, c'est l'énergie spirituelle, qui se distingue des autres formes d'énergie par la faculté qu'elle a de tirer d'ellemême plus qu'elle ne contient. Le triomphe de la vie culmine dans la créativité. L'œuvre que l'humain est appelé à réaliser est la création de soi par soi, la croissance de la personnalité par un effort qui tire beaucoup de peu et contribue à enrichir le monde.

4. H. Bergson, L'énergie spirituelle, Paris, P.U.F, 1967, p. 18. 


\subsubsection{L'affinité fondamentale selon Pierre Teilhard de Chardin}

À l'instar de Bergson, Teilhard de Chardin aborde la spiritualité dans le contexte de l'évolution de la vie. Toutefois, contrairement à son prédécesseur, il rejette l'hypothèse d'un univers entièrement formé de déterminismes, dans lequel se seraient développés, au hasard, des éléments spirituels spontanés. Il propose, au contraire, l'hypothèse d'un cosmos formé initialement de " libertés » élémentaires, d'un univers à étoffe spirituelle. S'appuyant constamment sur les connaissances issues de la biologie et de la physique, Teilhard de Chardin tente de prouver que toute l'histoire de l'évolution de l'univers n'est que le lent rassemblement d'une conscience diffuse, échappant graduellement aux conditions matérielles, pour répondre à une force qui l'attire vers le Centre. Au cours de l'évolution, l'émergence de la pensée a donné accès aux réalités morales et a marqué l'atteinte d'un niveau de conscience supérieur, où les forces physico-morales se ressentent comme une affinité fondamentale reliant les individus les uns aux autres et au Centre Oméga. Cette affinité fondamentale à laquelle se réduisent toutes les activités de l'univers, Teilhard de Chardin la nomme amour. Pour devenir pleinement humain, c'est-à-dire libre, l'être humain doit s'ouvrir à cette force d'attraction qu'est l'amour ${ }^{5}$.

\subsubsection{L'exigence de transcendance selon Gabriel Marcel}

C'est d'un point de vue phénoménologique que Gabriel Marcel aborde sa réflexion sur le mystère de l'être. Le questionnement sur la nature de l'être semble être universel et répond, selon le philosophe, à une exigence de transcendance. Cette exigence se ressent comme la certitude de ne pas être réduit à un ici, qui serait un lieu d'exil fortuit, par opposition à un certain Centre, qui est le lieu réel et qui ne peut être évoqué que comme un au-delà. Malgré tout ce qu'on peut donner comme preuve à l'humain qu'il est chargé de déterminismes, celui-ci se ressent comme un être libre. Phénoménologiquement, cette conscience d'être libre est une réalité. Ce qui permet à l'être humain de porter un jugement d'existence sur son moi sans le réduire à un état d'objet est le fait qu'il se perçoit comme un ego parmi d'autres avec lesquels il ressent "une communauté profondément enracinée dans l'ontologique, sans laquelle

5. P. TeIlHARD de Chardin, L'énergie humaine, Paris, Seuil, 1962. 
les liens humains seraient inintelligibles ${ }^{6} »$. Le « je » n'existe que dans un rapport intersubjectif, dans l'amour.

\subsubsection{L'ouverture au Tu fondamental selon Martin Buber}

Marcel reconnaît à Buber le mérite d'avoir étoffé cette notion d'intersubjectivité des ego. Pour résister au non-être, Buber affirme que les être humains doivent refouler les empiétements du Cela et s'engager dans la relation. Le Je-Cela constitue le monde de l'expérience alors que le $J e-T u$ fonde le monde de la relation. Le Cela se développe souvent au détriment de l'aptitude à la relation; il est voué à la disparition car il est régulé par des lois immuables, les lois de la matière et de l'évolution, contre lesquelles toute résistance est inutile. La rencontre de deux « Je » ne fonde toutefois pas la relation. Pour que celle-ci se produise, il faut s'ouvrir au Tu fondamental, au centre vivant qui relie les êtres vivants les uns aux autres dans les liens de vivante réciprocité. C'est cet acte d'ouverture qui donne vie à l'humanité au-delà du temps et de l'espace, qui connecte à ce vrai lieu dont parle Marcel, à cette demeure cosmique de l'homme ${ }^{7}$.

\subsubsection{La préoccupation ultime selon Paul Tillich.}

Tillich aborde la spiritualité dans une perspective existentielle qui fonde la foi sur le courage d'être, lequel permet d'affronter l'angoisse de l'existence. Il conçoit la spiritualité comme une démarche humaine fondamentale et universelle, une visée de la conscience vers la profondeur, vers un sens ultime. La préoccupation ultime est ce qui donne la direction et l'unité à toutes les autres préoccupations de sens et, par là, à la personnalité. Elle est le centre qui structure tous les éléments de la vie personnelle ; c'est l'élément intégrateur de la personnalité. S'engager dans la profondeur, c'est affronter courageusement la possibilité du non-être qui se ressent comme angoisse : l'angoisse de la fatalité et de la mort (angoisse ontologique), l'angoisse de l'absurde et du vide (angoisse spirituelle) et l'angoisse de la culpabilité et de la damnation (angoisse morale) ${ }^{8}$.

6. G. MARCEL, Foi et réalité, vol. 2, Paris, Aubier, 1951, p. 20.

7. M. BubER, Je-Tu / trad. par G. Bianchis, Paris, Aubier-Montaigne, 1969 (allemand 1923).

8. P. TilliCH, Le courage d'être / trad. par F. Chapey, Bruxelles, Casterman, 1967 (anglais 1952). 


\subsubsection{Vers une définition de la spiritualité}

Il ressort de ces différentes conceptions qu'il existerait une impulsion universelle vers la transcendance, vers des dimensions qui dépassent le moi, quelle que soit la forme que prend cette impulsion (élan vital, attraction fondamentale, exigence de transcendance, ouverture au Tu fondamental ou préoccupation ultime). La transcendance se ressent, sur un plan intrapersonnel, comme le sentiment de la présence en soi d'un centre intégrateur. Elle se vit en même temps sous un mode interpersonnel, comme le sentiment profond d'être intimement lié à la communauté humaine. Enfin, la transcendance ouvre à une expérience transpersonnelle, en faisant communier avec le Divin, l'Universel, l'Absolu, l'Ultime, quel que soit le nom donné à cette force. Par ailleurs, deux fonctions de la spiritualité se dégagent de ces thèses : une fonction de sens, la spiritualité permettant d'échapper au non être et d'intégrer les expériences énigmatiques de la vie dans un cadre de sens qui maintient l'espoir, et une fonction de croissance, qui donne l'élan vers la pleine actualisation du potentiel humain.

À la suite de cette synthèse, une définition de la spiritualité a été proposée : La spiritualité est une aspiration vers la transcendance en vue d'intégrer les différentes facettes de l'expérience humaine dans un tout cohérent et d'actualiser toutes les potentialités de croissance.

\subsection{Croissance et spiritualité : perspectives issues des sciences bu- maines et des sciences de la santé}

La revue des écrits provenant des sciences humaines et de la santé soutient l'émergence d'une transcendance comme caractéristique du développement humain. Selon les approches dites "maturationnelles ", le développement suit un schéma universel, ordonné, qui progresse par étapes hiérarchiques. Selon ces théories, l’organisme a un plan de développement déterminé qui peut être actualisé lorsque les interactions avec le milieu sont favorables. Toutes les potentialités de croissance existent au départ et se développent progressivement en fonction du stade de développement. La croissance procède par étapes de plus en plus complexes au fur et à mesure que de nouvelles habiletés organiques émergent. La transcendance apparaît comme un point culminant du développement. 
D'autres approches, plus " contextuelles ", avancent que le développement suit une trajectoire individuelle et unique, marquée par des accidents de parcours qui en influencent la direction. Ces facteurs contextuels créent une rupture dans le synchronisme des différentes dimensions biologique, psychologique, sociale, culturelle et environnementale qui constituent l'expérience humaine. Le désordre qu'engendre cette rupture est porteur de changement en ce qu'il favorise l'émergence de nouvelles possibilités pour rétablir le synchronisme et le sens. Dans ces approches, la transcendance est une option de croissance que l'individu est libre de choisir en vue de résoudre les contradictions entre ses dimensions conflictuelles.

\subsubsection{Facteurs maturationnels, intégration et transcendance}

Jung est un des premiers psychanalystes à avoir considéré le développement de la personnalité de la naissance à la vieillesse. Il affirme que pour devenir pleinement autonome et intégré, l'humain doit cheminer dans son processus d'individuation qui le conduit du moi au Soi. L'individu qui n'acquiert pas les caractéristiques de la connaissance de soi à une étape de son développement en retarde la progression. La vieillesse est l'étape de l'intégration de sa spiritualité. En effet, c'est en se connaissant mieux que l'individu âgé peut atténuer la couche d'inconscient personnel qui voile l'inconscient collectif. En atteignant cet inconscient, l'humain transcende les limites du temps et de l'espace et prend conscience de sa relation à l'humanité depuis le début des temps et pour l'éternité. L'ouverture au Soi révèle l'image du divin en chaque individu, le faisant accéder à un ordre qui le dépasse ${ }^{9}$.

Si Jung a eu le mérite de proposer une vue du développement qui s'étend sur toute la longueur de la vie, c'est à Erikson que l'on doit la première théorie psychanalytique qui décrit plus spécifiquement le développement adulte. Selon sa théorie, le développement comporte huit étapes hiérarchiques, chacune présentant deux tendances opposées. Pour poursuivre sa croissance, l'individu doit résoudre la crise de croissance en réussissant à faire pencher la balance vers le pôle positif. Chaque crise non résolue ralentit le développement. La résolution d'une crise se solde par l'acquisition d'une vertu. Durant la vieillesse, les

9. C.G. Jung, Modern Man in Search of a Soul, New York, Harcourt, Brace et Jovanovich, 1933. 
pôles à intégrer pour accéder à la vertu de sagesse sont l'intégrité du moi et le désespoir. L'intégrité du moi est un état d'esprit qui se traduit par un amour de l'ego humain, lequel infuse un ordre et un sens spirituel au monde et conduit à accepter sa vie comme ayant contribué significativement à cet ordre. Erikson affirme que le sens du «Je » dans la vieillesse a une dernière occasion de transcender les limites du temps et de l'espace pour s'ouvrir à une identité intersubjective, un sens du "Nous » dont la personne âgée peut devenir un témoignage vivant ${ }^{10}$.

Plusieurs théoriciens de l'intelligence ont reconnu la présence chez l'adulte de stades de développement de la pensée qui vont au-delà du stade piagétien de la pensée formelle. Ces auteurs ont mis en évidence un processus développemental de la pensée propre à l'adulte, le niveau post-formel. Dans cette lignée, Labouvie-Vief considère que l'adulte qui a atteint le niveau post-formel établit une interaction dynamique entre deux modes de connaissances, le logos et le mythos. Le logos constitue la connaissance objective, rationnelle, mécanique et précise. Le mythos est la connaissance subjective, imaginative, émotive. Le mythos est le premier mode de connaissance que l'enfant expérimente ; par la suite, il se ferme progressivement à son expérience intérieure pour mieux saisir le monde extérieur et le catégoriser de manière fiable et sécurisante. Selon Labouvie-Vief, la tâche de l'adulte est de réintégrer le mythos en assouplissant la rigueur du logos, car l'adulte qui analyse la réalité avec les seules opérations formelles de la pensée logique est incapable de résoudre certains problèmes liés au vieillissement. En réintégrant le mythos, l'adulte vieillissant peut donner un sens aux contradictions qui se multiplient dans sa vie en faisant une place à la profondeur émotionnelle et aux préoccupations spirituelles. La sagesse qui résulte de l'intégration des deux modes de connaissance permet à l'individu de maintenir la tension paradoxale entre une pensée à la fois immanente et transcendante ${ }^{11}$.

10. E.H. ERIKSON, Childhood and Society, New York, Norton, 1963 (1950). 11. G. LabouviE-VIEF, "Modes of Knowledge and the Organization of Development ", dans M.L. Commons et al., dir., Adult Development, vol. 2, New York, Preager, 1989, p. 43-62 ; R.T. STERnberG, "Wisdom as integrated Thought. Historical and Developmental Perspectives ", dans Wisdom : Its Nature, Origins, and Development, Cambridge, Cambridge University Press, 1990, p. 52-83. 
Dans ces théories provenant de la psychologie analytique, de la psychanalyse et de la psychologie cognitive, la fonction de croissance de la transcendance est mise de l'avant. Elle est inscrite dans les possibilités du développement organique dont elle constitue l'étape ultime. En ce sens, ces auteurs se rapprochent des hypothèses de Bergson et de Teilhard de Chardin. Aussi, comme dans les thèses de ces deux auteurs, la transcendance est l'aboutissement d'un processus intrapersonnel qui se vit sous un mode interpersonnel et ouvre au transpersonnel. Toutefois, la croissance peut être ralentie ou compromise si la personne échoue à intégrer les nouvelles caractéristiques à une étape de son développement, de sorte que la pleine réalisation du potentiel humain n'est accessible qu'à une minorité d'individus.

\subsubsection{Facteurs contextuels, recherche de sens et transcendance}

Pour les théoriciens qui considèrent le désordre comme le moteur du changement, le développement suit une trajectoire peu prévisible, qui fait moins de place à la continuité et à l'accumulation. L'accent est mis sur la capacité individuelle de donner un sens au désordre pour poursuivre sa croissance.

Frankl soutient plusieurs postulats qui font de lui un précurseur de cette école. Psychiatre juif ayant vécu l'expérience des camps nazis, il a pu constater que les besoins supérieurs de croissance peuvent être satisfaits alors même que la survie et la sécurité sont menacées. Frankl conteste donc l'hypothèse d'une hiérarchie des besoins, telle que proposée par Maslow, et, par là, d'un principe directeur d'actualisation de soi. Il affirme que la réalisation de la nature humaine passe par l'auto-transcendance et l'oubli de soi plutôt que par l'actualisation de soi. Selon lui, il existerait en chaque être humain un inconscient spirituel, lequel se présente comme une volonté de sens, que chacun est libre d'accepter ou de refuser. Cette volonté crée une tension qui pousse l'individu à faire des choix qui peuvent contribuer à rendre sa vie signifiante ou vide de sens. En actualisant le sens potentiel des situations rencontrées dans sa vie, la personne progresse vers l'autotranscendance et se rapproche de la conscience d'un sens profond. Bien que son approche se situe dans une perspective existentielle (sens dans la vie), Frankl soutient qu'un sens dernier, un supra-sens (sens de la vie), peut être découvert. Ce but ultime vers lequel pointe naturellement l'inconscient est Dieu. Ce Dieu n'est celui d'aucune religion et 
il appartient à chaque individu de trouver le symbole qui exprimera le mieux le supra-sens de sa vie ${ }^{12}$.

Dans le domaine du développement cognitif, Riegel avance que la croissance est le résultat du dialogue unique et réciproque entre toutes les dimensions de l'expérience humaine : la dimension biologiqueinterne, la dimension psychologique-interne, la dimension physiqueexterne et la dimension sociale-culturelle. L'absence de synchronisme entre deux ou plusieurs dimensions crée une tension d'où peut surgir le changement. Selon Riegel, la vieillesse est une étape propice au changement car les conflits entre les dimensions sont nombreux. Pour résoudre la contradiction, l'individu doit procéder à une réinterprétation qui rétablit le synchronisme. Riegel ajoute que lorsque la réinterprétation permet une synchronisation prospective, alors même les incapacités et la mort peuvent prendre un sens ${ }^{13}$.

Ces théories qui donnent préséance au sens rejoignent les nouvelles théories infirmières de l'être humain à la poursuite de sa santé. La théorie de l'Être-en-devenir de Parse est fondée sur trois concepts clés : le sens, la rythmicité et la transcendance. Le sens est continuellement construit par chaque individu à tous les niveaux de l'univers, créant la réalité du moment et révélant peu à peu le sens ultime de la vie. La santé est "l'être-en-devenir » qui se co-constitue avec l'univers dans son unicité et sa diversité, à travers des patrons paradoxaux de relations que Parse conçoit comme des processus rythmiques naturels de la vie. Ainsi, par les choix qu'il pose, l'individu se rapproche de personnes, d'idées et d'objets en même temps qu'il s'éloigne d'autres personnes, d'autres idées et d'autres objets (connecting/separating). Chaque situation offre des occasions de développer des habiletés tout en confrontant à des limites (enabling/limiting), révélant une partie de soi en même temps qu'elle en voile une autre (revealing/concealing). La personne et son environnement évoluent simultanément au rythme de ces relations paradoxales, en co-transcendant selon les possibilités du moment dans un processus continu de transformation qui conduit vers de nouvelles possibilités ${ }^{14}$.

12. V.E. FRANKL, Man's Search for Meaning. An Introduction to Logotherapy, Boston, Beacon Press, 1963.

13. K.F. RIEGEL, Foundations of Dialectical Psychology, New York, Academic Press, 1979. 
Adoptant une approche contextualiste du développement, Reed, une autre infirmière, propose une théorie de l'auto-transcendance. Elle affirme que la quête de sens fait partie intégrante de la démarche de développement et de maintien de la santé. La personne et l'environnement interagissent dans une dynamique qui transforme en énergie les conflits et les défis inhérents à la vie. Reed soutient que l'autotrancendance est un élément-clé dans le processus de changement. La maladie et la vieillesse sont des situations qui font ressentir cruellement les limites du corps, du temps et de l'espace. Le dialogue entre la personne et son contexte engendre alors des conflits qui peuvent donner l'impulsion vers le développement d'une forme d'autotranscendance ${ }^{15}$. Reed définit la spiritualité comme la propension à faire du sens par le sentiment d'être intimement en relation avec des dimensions qui dépassent le soi, et ce, d'une façon qui transforme et rend plus fort. La transcendance peut se vivre au plan intrapersonnel (sentiment d'une interconnexion qui se ressent en soi), au niveau interpersonnel (sentiment d'être lié aux autres et à la nature) et transpersonnel (sentiment d'être lié à l'Invisible) ${ }^{16}$.

Ces théories issues de la psychiatrie, de la psychologie cognitive et des sciences infirmières mettent l'accent sur la fonction de sens de la spiritualité. Selon ces auteurs, l'ouverture à une perspective transcendantale est une option de sens que chacun est libre de choisir. Ces thèses rejoignent celles de Buber, de Marcel et de Tillich. La liberté de choix, la responsabilité de ses choix et la dimension intersubjective y structurent l'expérience d'être et de croître des individus. La transcendance se ressent aussi aux niveaux intra-, inter-, et transpersonnel. Par ailleurs, le développement respecte un tracé unique et peu prévisible qui libère un potentiel de croissance à tout âge, quel que soit le niveau de développement antérieur.

14. R.R. PARSE, " Human Becoming. Parse's Theory of Nursing ", Nursing Science Quarterly, 5 (1992) p. 35-42.

15. P. G. REED, "Toward a Nursing Theory of Self-Transcendence. Deductive Reformulation Using Developmental Theories ", Advances in Nursing Science, 13 (1991) p. 64-77.

16. P. G. REED, « An Emerging Paradigm for the Investigation of Spirituality in Nursing ", Research in Nursing and Health, 15 (1992) p. 349-357. 


\subsection{Constat}

Le contraste entre l'abondance des écrits théoriques qui suggèrent l'hypothèse d'une croissance durant la vieillesse dont l'essence serait de nature spirituelle et la pauvreté de la recherche pour en vérifier l'existence est remarquable. Le rejet, au début du siècle dernier, de l'expression traditionnelle de la transcendance par les intellectuels et les scientifiques, l'exacerbation de l'angoisse existentielle, la victimisation de la vieillesse et la gérontophobie qui en a découlé, ainsi que l'hégémonie du paradigme positiviste en sciences, comptent sans doute parmi les facteurs socio-historiques qui ont freiné le développement des connaissances empiriques sur ce phénomène ${ }^{17}$. C'est à ces connaissances que la présente étude voulait contribuer.

\section{2. Étude phénoménologique}

\subsection{Méthode}

Afin de recruter des personnes âgées vivant plusieurs déclins, la recherche a été conduite dans des centres d'hébergement et de soins de longue durée (CHSLD). Quatre centres ont accepté de collaborer au projet. Des entrevues dans un centre de jour ont aussi été réalisées afin de recueillir des informations sur le phénomène alors que les déclins ne bouleversent pas complètement le mode de vie.

Les responsables des centres ont établi une liste de personnes satisfaisant aux critères d'inclusion, lesquels étaient : être âgé de soixantedix ans et plus, être mentalement compétent, parler français, pouvoir discourir facilement, avoir un état de santé qui permette de supporter une entrevue de soixante minutes.

Vingt-deux personnes ont accepté de participer à la recherche, dont seize ont rapporté une expérience de croissance. Dix d'entre elles discouraient facilement sur leur expérience. Elles forment l'échantillon sur lequel s'appuie principalement l'analyse des données. Six autres person-

17. H.R. Moody, "Toward a Critical Gerontology. The Contribution of the Humanities to Theories of Aging ", dans J.E. BIRREN et V. L. BENGTSTON, dir., Emergent Theories of Aging, New York, Springer Publishing Co., 1988, p.19-39 ; L. TORnSTAM, "The Quo Vadis of Gerontology. On the Scientific Paradigm of Gerontology », The Gerontologist, 32 (1992) p. 318-326. 
nes ont dit vivre de la croissance tout en ayant de la difficulté à élaborer leur pensée sur le phénomène ; les données issues de leurs entrevues ont été utilisées pour enrichir les résultats de l'analyse des données. Enfin, six personnes ont raconté une expérience de déclin. Les données issues de leurs entrevues soutiennent les résultats de l'analyse des données en utilisant la méthode de comparaison avec les cas contraires.

L'âge des dix personnes formant l'échantillon principal varie de soixante-dix à quatre-vingt-onze ans. En ce qui concerne le sexe, il y a une prédominance féminine (sept femmes, trois hommes). Toutes les personnes qui forment l'échantillon principal vivent seules, contrairement à celles des autres groupes où l'on observe la participation d'individus vivant avec un conjoint. Cette solitude est un facteur qui semble favoriser une plus grande conscience de l'expérience de croissance et peut expliquer que ces personnes pouvaient la décrire plus précisément. Tous les participants de l'échantillon principal proviennent d'un CHSLD alors que sept des douze autres participants vivent encore à domicile. En ce qui a trait à la scolarité, la répartition égale entre les individus peu scolarisés et les plus scolarisés assure que l'échantillon n'est pas élitiste. Il y a une nette prédominance de l'identité canadienne-française (neuf sujets) alors que l'identité religieuse est majoritairement catholique (sept sujets).

Toutes les personnes qui forment l'échantillon principal se sont dites très croyantes. Leur parcours religieux est toutefois plus diversifié que celui des participants des deux autres groupes. Parmi les sept personnes qui sont de confession catholique romaine, deux intègrent l'idée de réincarnation à leurs croyances et une est en rupture avec les autorités religieuses sans qu'aucune n'en ressente un inconfort religieux. En ce qui concerne les trois autres répondants, deux ont quitté les rangs de la religion catholique pour se joindre à des mouvements sectaires, et un autre, considérant que Dieu est le même pour tous, préfère dire qu'il est de religion universelle.

La question adressée aux répondants était précédée d'un préambule dont l'objectif était de diminuer un effet possible de conformité sociale :

Certaines personnes décrivent la vieillesse comme une série de pertes et de déclins alors que d'autres pensent qu'elle comporte aussi des gains ou des occasions de croître, de se développer. Cette perception dépend de l'expérience de chacun, de sorte qu'il n'y a pas de bonne ou de mauvaise 
réponse. Dans votre cas, diriez-vous que la vieillesse est surtout une période de déclins ou qu'elle comporte aussi des occasions de croissance?

Pour les personnes qui disaient vivre de la croissance, la question phénoménologique était : Comment décririez-vous cette croissance? Alors que pour les personnes qui disaient vivre surtout du déclin, la question était : Parlez-moi de votre expérience de la vieillesse.

En vue de dégager les caractéristiques essentielles et la structure du phénomène de croissance, les données ont été analysées selon les étapes suivantes : 1) transcription verbatim de chaque entrevue ; 2 ) lecture attentive de chaque entrevue pour saisir les données pertinentes au phénomène ; 3 ) reconstitution du récit en éliminant les phrases non pertinentes ou répétitives ; 4) division du récit en unités de signification ; 5) approfondissement du sens des unités de signification en les traduisant dans le langage des sciences humaines ; 6) synthèse et intégration des unités de signification transformées en une description de la structure typique individuelle du phénomène ; 7) synthèse des structures individuelles en une structure générale typique du phénomène. Les résultats ont été soumis à des juges à différentes étapes du processus d'analyse.

\subsection{Résultats}

L'identification des essences fondamentales de l'expérience de croître à travers les déclins en vieillissant donne la réponse à la première question de la recherche: Quelles sont les caractéristiques essentielles de l'expérience de croissance de personnes âgées vivant plusieurs déclins ? L'analyse des données issues des entrevues auprès des personnes qui ont dit vivre une croissance a permis d'isoler six thèmes fondamentaux autour desquels se structure l'expérience de croissance durant la vieillesse : accepter délibérément les pertes, affirmer sa force, accueillir sereinement la mort, apprécier plus intensément la vie, s'engager positivement dans sa solitude et enrichir son univers relationnel. Quatorze thèmes secondaires leur sont reliés. Ces thèmes essentiels dialoguent deux à deux dans une logique de perte et de gain, laissant deviner que la croissance durant la vieillesse réside dans l'habileté de la personne âgée à réaliser un gain à partir d'une perte (voir tableau 1 ). 
T A B LEA U 1

\section{Dialogue des thèmes}

\begin{tabular}{|l|l|}
\hline $\begin{array}{l}\text { Choisir une attitude positive } \\
\text { en donnant un sens à la perte }\end{array}$ & Découvrir et réaliser le gain \\
\hline $\begin{array}{l}\text { Accepter délibérément les pertes } \\
\text { - faire preuve de réalisme et de maturité } \\
\text { - avoir des raisons d'accepter }\end{array}$ & $\begin{array}{l}\text { Affirmer sa force } \\
\text { - démontrer sa détermination et son courage } \\
\text { - rester maître de sa vie }\end{array}$ \\
\hline $\begin{array}{l}\text { Accueillir sereinement la mort } \\
\text { - être prêt(e) à mourir en tout temps } \\
\text { - avoir des explications satisfaisantes }\end{array}$ & $\begin{array}{l}\text { Apprécier plus intensément la vie } \\
\text { - goûter le moment présent } \\
\text { - aimer et se sentir aimé(e) } \\
\text { - demeurer dans le coup }\end{array}$ \\
\hline $\begin{array}{l}\text { S'engager positivement dans sa solitude } \\
\text { - identifier les bienfaits de la solitude } \\
\text { - questionner ou approfondir ses croyances }\end{array}$ & $\begin{array}{l}\text { Enrichir son univers relationnel } \\
\text { - se rapprocher de l'autre } \\
\text { - se rapprocher de la nature } \\
\text { - se rapprocher du Divin }\end{array}$ \\
\hline
\end{tabular}

Dans les thèmes manifestant une attitude de croissance, les collaborateurs s'appuient à la fois sur la pensée rationnelle et pragmatique pour donner un sens aux pertes (faire preuve de réalisme et de maturité, être prêt à mourir en tout temps, identifier les bienfaits de la solitude) et sur la pensée symbolique et mythique, en cherchant dans leurs croyances des explications qui donnent un sens à cette perte (avoir des raisons d'accepter, avoir des explications satisfaisantes, questionner ou approfondir ses croyances). Une fois que le sens est donné, le gain se révèle et est actualisé par la personne âgée en croissance. Ainsi, l'expérience de croître à travers les déclins durant la vieillesse se livre finalement sous trois essences fondamentales paradoxales :

1) Faire l'expérience d'accepter délibérément les pertes en même temps qu'affirmer sa force

C'que l'bon Dieu fait, j'Lui reproche pas. Je l'accepte...J'accepte mais j'me laisse pas aller. Faut mettre du piquant, faut avoir du cœur au ventre. Y'a été un temps, j'parlais moins que ça...J'me suis pris en main, pis me v'là aujourd'hui. [monsieur Charles]

De l'avis de tous les collaborateurs qui ont dit vivre de la croissance, il n'y a pas de croissance possible sans cette acceptation volontaire des pertes. La décision d'accepter est la pierre angulaire de l'expérience de croissance durant la vieillesse. C'est l'épreuve initiatique, celle qui ouvre la porte à toutes les possibilités de croissance. Vieillir sollicite sans cesse la capacité d'accepter d'une personne dont les ressources 
physiques, psychologiques et sociales sont affaiblies. Le défi de cette acceptation prend alors tout son sens. C'est d'abord en invoquant le principe de réalité et de bon sens que les aînés font le choix d'accepter. Constatant qu'ils ne peuvent se soustraire aux pertes et considérant que le refus ne ferait que les rendre plus malheureux, ils optent pour l'acceptation et trouvent des justifications qui facilitent leur choix : être satisfait de sa vie et en avoir profité en temps et lieu, faire confiance au dessein de Dieu...

Une fois l'acceptation faite, le gain se révèle. Dans tous les récits transparaît cette fierté d'avoir réussi une épreuve difficile. Ces personnes qui vivent de la croissance sont conscientes que la force nécessaire pour accepter n'est pas à la portée de tous et qu'elles font partie d'une élite. Les mots qu'elles choisissent pour exprimer leur victoire sur la résignation et le découragement traduisent la perception positive qu'elles ont d'elles-mêmes. Il faut " avoir beaucoup de vouloir ", " se prendre en main ", " se parler fort ", " ne pas s'écouter ", " ne pas lâcher ", " avoir du cœur au ventre " "vaincre le négatif et la peur ». C'est en communiquant les preuves de leur ténacité et de leur courage, en réussissant l'exploit de rester maîtres d'une vie dont le contrôle paraît pourtant leur échapper, que les collaborateurs ont démontré leur force.

\section{2) Faire l'expérience d'accueillir sereinement la mort en même temps qu'apprécier plus intensément la vie}

La mort ne me fait pas peur. C'est la fin de ce que je suis mais ce qui arrive à l'âme, je l'sais pas. J'm'intéresse plus à la vie. J'me sens bien. J'suis heureuse. J'aime pas quand quelqu'un dit : "c'est triste la vie ». La vie, quand on veut, est pas triste. Y'a des épreuves mais dans l'ensemble, c'est beau la vie. C'est un don de Dieu. [madame Laura]

Accueillir sereinement la mort est la suite logique de l'acceptation des pertes. Avec les déclins qui s'accumulent, la mort se fait omniprésente. Les personnes âgées en croissance lui font face sans crainte et l'accueillent même avec indifférence. Elles se sont préparées, ont réglé leurs affaires de façon à pouvoir partir en tout temps sans inquiétude et se sont données des explications satisfaisantes sur ce qui arrive après la mort. Plutôt que de perdre le précieux temps qui reste à regarder venir la mort, c'est plutôt vers la vie que se tourne leur regard.

Tous les témoignages manifestent, en effet, une étonnante soif de vivre. Déjà, en prenant le parti d'accepter les pertes, les répondants 
ont acquis l'habileté de diriger leur regard sur ce qui est, plutôt que sur ce qui n'est plus. Mettant à profit ce gain, ils découvrent que la vie renferme encore des promesses. "La vie, j'la vois belle ", dira monsieur Charles, en dépit d'un deuxième accident vasculaire cérébral qui lui a fait perdre tout le chemin parcouru dans sa réadaptation après le premier épisode. La célébration de leur vie, qui apparaît objectivement assez terne, est un des aspects les plus surprenants et les plus émouvants des récits. En disant oui à la vie, ces aînés découvrent des possibilités que d'autres sont incapables de voir. Ils apprennent à goûter le moment présent, continuent d'aimer et de se sentir aimés(es), et demeurent dans le coup jusqu'au bout.

3) Faire l'expérience de s'engager positivement dans sa solitude en même temps qu'enrichir son univers relationnel

Y'a des pertes en vieillissant mais y'a un gain enrichissant. Moi, j'ai la foi. Ma foi est différente aujourd'hui. Est plus profonde...Avant j'pensais moins à Dieu, j'avais pas l'temps...Aujourd'hui, j'ai le temps de lire la Bible. J'lis les psaumes avec attention pour mieux m'approcher de Dieu... Avec les autres, j'ai pas le même tempérament. J'suis plus à l'écoute. Avant, j'me fichais des autres, j'pensais à moi premièrement, c'est tout. [madame Laurette]

Le thème s'engager positivement dans sa solitude démontre que l'activité réflexive est au cœur de l'expérience de croissance à travers les déclins. Plus les pertes s'accumulent, plus les personnes âgées pénètrent dans la solitude. Celles qui vivent de la croissance tirent profit de ces nombreux moments pour questionner et approfondir leurs croyances. Elles recherchent dans l'ordre du transcendant des réponses qui pourraient donner un sens à ce qu'elles vivent et rendre plus cohérentes leurs expériences présentes.

Cette réflexion les conduit à être plus conscientes de leur univers relationnel. Un premier pas dans la compréhension de leur expérience relationnelle leur fait découvrir autrui. Toutes se disent plus sages dans leurs relations, plus solidaires et plus altruistes. En regardant autour d'elles, plusieurs découvrent la nature et prennent conscience de cette relation étroite qui les lie à leur environnement. Enfin, à mesure qu'elles s'engagent dans leur solitude, les personnes âgées qui vivent de la croissance lèvent le voile sur le chemin qui les conduit à Dieu. Ainsi, en même temps que leur vie sociale se rétrécit, elles se rapprochent de l'autre, de la nature et du Divin. 
L'identification de ces caractéristiques essentielles dévoile la structure typique sous-jacente à ce phénomène de croissance et donne la réponse à la deuxième question de la recherche : Quelle est la structure générale de l'expérience de croissance de personnes âgées vivant plusieurs déclins ? Confrontée aux pertes, à celles qu'elle vit déjà et à celles qui se profilent à l'horizon, la personne âgée qui vit de la croissance s'engage résolument à relever les défis de sens que lui pose son existence, de façon à transcender les pertes. Cette démarche la conduit à accepter délibérément les pertes tout en affirmant sa force, à accueillir sereinement la mort tout en appréciant plus intensément la vie et à s'engager positivement dans sa solitude tout en enrichissant son univers relationnel.

Enfin, pour répondre à la troisième question de la recherche : Quelle part la spiritualité occupe-t-elle dans cette expérience de croissance de personnes âgées vivant plusieurs déclins? il faut retourner à la définition de la spiritualité qui a été proposée au début de cet exposé : " la spiritualité est une aspiration vers la transcendance en vue d'intégrer les différentes facettes de l'expérience humaine dans un tout cohérent et d'actualiser toutes les potentialités de croissance ». L'analyse des données confirme que l'expérience de croissance de ces personnes âgées est à plusieurs égards une expérience de transcendance qui donne un sens à leur vécu et contribue à la poursuite de leur développement :

- Pour accepter délibérément les pertes tout en affirmant sa force, les personnes interrogées ont témoigné qu'elles avaient dû réaliser un premier niveau de transcendance, une transcendance corporelle, laquelle, en favorisant un détachement du corps et de ses contraintes, leur a permis de déplacer leur regard de la perte vers le gain.

J'm'arrange pour que ça aille bien. J'vois le bon côté des choses. J'ai perdu une jambe pis un bras mais j'ai gardé ma tête. Alors j'essaie de m'en servir le plus possible [rires] [monsieur Louis]

- Accueillir sereinement la mort tout en appréciant plus intensément la vie se réalise au terme d'une transcendance temporelle, faisant en sorte que nos répondants inscrivent naturellement leur histoire personnelle, dont ils sont fiers, dans l'histoire plus large de l'humanité, donnant ainsi sens à un présent qui apparaît pourtant insensé aux yeux des observateurs.

J'pense souvent à ma jeunesse, à ma vie, et j'ai pas de regret. J'suis satisfait de ma vie...J'suis content de m'être rendu là pis j'suis prêt pour 
l'autre étape. J'ai pas peur de mourir. Je crois que la mort c'est la fin de l'individu, qu'y a pas d'autres vies après. Par contre, j'crois pas qu'on meurt comme espèce, j'crois pas à la fin du monde. C'est comme ça qu'on continue à vivre. Y'a un plan directeur, la vie n'est pas venue de rien. Y'a Dieu au départ, mais Son plan c'est pas pour moi, c'est pour l'espèce. Moi, ma contribution, j'la donne de mon vivant. On est responsable de nos actes pour ceux qui nous suivent. [monsieur Georges]

- Enfin, l'expérience de s'engager positivement dans sa solitude tout en enrichissant son univers relationnel les fait accéder à cette transcendance décrite par les philosophes et les théologiens, laquelle se ressent sous un mode intrapersonnel comme la présence en soi d'un centre intégrateur, sous un mode interpersonnel comme le sentiment d'être lié aux autres ou à la nature et sous un mode transpersonnel comme une communion avec l'Infini.

Le tableau 2 reprend la définition de la spiritualité et démontre, à partir des témoignages des répondants âgés, que la spiritualité est au cœur de leur expérience de croissance.

TA B LEA U 2

La spiritualité est une aspiration vers la transcendance

transcendance intrapersonnelle

«Pour moi, la solitude c'est positif. Ce que j'appelais plus jeune « l'ennui », ça n'existe plus, dans le sens qu'on est jamais seule quand on a une vie intérieure bien remplie. L'ennui est remplacé par une présence intérieure... Cette présence, C'est moi tout en étant pas moi, comme un trésor dont j’peux jouir mais dont je ne tire pas les ficelles. » [madame Marie]

\section{transcendance interpersonnelle}

"Quand on vit avec les autres, comme ici, on a beaucoup à apprendre d'eux. J'apprends à plus écouter....J'apprends tous les jours...Aider les autres, c'est ce qui est le plus important pour moi pour les années qu'il me reste à vivre. J'me dis qu'on est sur la terre pour être ensemble. Faut embarquer avec les autres. » [madame Sylviane]

« Mon idée de Dieu, c'est que c'est un être Infini que je sens dans la nature. J'ai choisi ma chambre du côté du bord de l'eau. Je vois couler la rivière...La nature, c'est important. Il faut que je sois en contact avec la nature. » [madame Laura] 
TABLEAU 2 ( S U ITE)

transcendance transpersonnelle

«Ma conception de Dieu a changé. Dieu, c'est un amour qui m’entoure. J'Le sens près de moi. J'Lui parle comme à un ami. » [madame Laurette].

en vue d'intégrer les différentes facettes de l'expérience humaine dans un tout cohérent

«C'est pour ça que, avant d'arriver là (éternité), les souffrances que vous avez sur la terre vous aident à être plus avancé. Mais on a la volonté libre, on est tous Adam et Ève... Le Bon Dieu ne peut pas forcer notre amour...Il nous a donné l'intelligence et la liberté de faire le choix. Alors, il faut essayer de faire les choix qui sont bons pour nous. Il faut suivre notre intuition. On pense que c'est notre intuition, mais c'est la voix de la Providence qui nous guide. " [madame Berthe]

et d'actualiser toutes les potentialités de croissance.

"Y'en a des choses à apprendre! Alors y faut pas s'arrêter, y faut continuer à se développer. Faut avoir les yeux ouverts et voir ce qui se présente à soi. C'est pour ça, c'est par curiosité que je tiens à vivre [rires] ! C'est plus pour moi-même que pour les autres, pour voir jusqu'où ça mène ce voyage-là. Parfois j'ai des doutes, mes choix sont plus difficiles. J'me dis ça me sert à quoi de tant travailler, tant d'efforts ! Pis tant pis ! Je r'commence à travailler. Pourquoi ? J'me dis que j'vais peut-être mourir demain, peut-être pas, mais il ne sera pas dit que j'me serai arrêtée en chemin. » [madame Marie].

En somme, la réponse à la troisième question de la recherche est que l'expérience de croître à travers les déclins durant la vieillesse constitue un défi spirituel avant tout. L'étude a démontré que la capacité humaine de créer du sens à travers le non sens est illimitée lorsqu'elle ouvre à la transcendance. 


\section{RÉSUMÉ}

Malgré nombre d'écrits théoriques soutenant l'hypothèse d'une croissance durant la vieillesse dont le substrat serait de nature spirituelle, peu de chercheurs s'intéressent à ce phénomène. Une étude phénoménologique, conduite auprès de vingt-deux personnes âgées de plus de 70 ans et vivant en centre d'hébergement, a établi que la croissance à travers les déclins durant la vieillesse se caractérise par trois expériences paradoxales : accepter délibérément les pertes en même temps qu'affirmer sa force, accueillir sereinement la mort en même temps qu'apprécier plus intensément la vie et s'engager positivement dans sa solitude en même temps qu'enrichir son univers relationnel. Les récits démontrent que le phénomène de croissance à travers les déclins durant la vieillesse se fonde principalement sur une expérience de transcendance faisant en sorte que cette croissance devient un défi spirituel avant tout.

\section{ABSTRACT}

Numerous thinkers have hypothesized that growth continues with aging and that this growth is of a spiritual nature. Few researchers however, have attempted to understand this phenomenon from an empirical perspective. This phenomenological study was conducted with twenty-two elderly persons, aged over 70 years and living in long-term care facilities. The purpose of this study was to describe the experience of growth with respect to the losses that are inevitable during aging. It was found that this experience is characterized by three paradoxes: to accept the losses while affirming one's strength; to welcome death with serenity while appreciating life more intensively; to engage positively in one's solitude while enriching one's relational world. The stories demonstrated that the phenomenon of growth with respect to the losses that are inevitable during aging, is founded mainly in the experience of transcendence. Hence, this growth becomes a spiritual challenge. 